\title{
MATLAB Tools for Linear and Nonlinear System Stability Theorem Implementation
}

\author{
James H. Taylor \& Cheney Chan \\ Department of Electrical and Computer Engineering \\ University of New Brunswick \\ PO Box 4400, Fredericton, NB CANADA E3B 5A3 \\ email: jtaylor@unb.ca
}

\begin{abstract}
Three MATLAB-based tools have been developed for the convenient assessment of stability conditions for linear and nonlinear systems. The first is a more helpful and definitive version of nyquist, which has features of automatic zooming (to show all crossings of the real axis and thus display the corresponding critical gain points) and which displays on the real axis a numeral (' 0 ', ' 1 ' etc.) that represents the number of unstable (right-half plane) poles that will occur in a closed-loop system with feedback gain $k$ if the point $-1 / k$ is located in that region. The second tool is built on this extended nyquist command, and makes the application of the nonlinear time-varying system Circle Criterion equally simple and definitive. Finally, a Popov Criterion tool is included to achieve better sector bounds for the nonlinear timeinvariant case. Examples are presented to show the efficacy of these extensions within the MATLAB environment.
\end{abstract}

\section{Introduction}

Existing MATLAB commands for the stability analysis of linear systems are not easy to interpret by the lessthan-expert user, and tools for assessing the stability of nonlinear systems are nonexistent in MATLAB. In the linear case, interpreting the usual MATLAB Nyquist plot is not hard if the plant is stable and the $W(j \omega)$ locus is not complicated, but it may be confusing, especially if there are multiple real-axis crossings and/or open-loop poles on the imaginary axis or in the righthalf plane (RHP). In the nonlinear case, none of the classical absolute stability criteria are implemented in MATLAB, and we have addressed that lack by creating comparably user-friendly tools for the application of the Circle Criterion for the time-varying case and the Popov Criterion for time-invariant nonlinear systems $[1,2,3]$.

\section{Stability Criteria}

In this section we state the linear and nonlinear stability criteria, emphasizing their classical geometric interpretations. As a preliminary, the class of systems considered is depicted in Fig. 1, where it is assumed that the forward-path transfer function is expressed in state-space form as:

$$
W(s)=C(s I-A)^{-1} B+D
$$

or in Laplace notation as a ratio of polynomials, with the order of the numerator not exceeding that of the denominator:

$$
W(s)=\frac{b_{m} s^{m}+\ldots+b_{1} s+b_{0}}{s^{n}+a_{n-1} s^{n-1}+\ldots a_{1} s+a_{0}}, m \leq n
$$

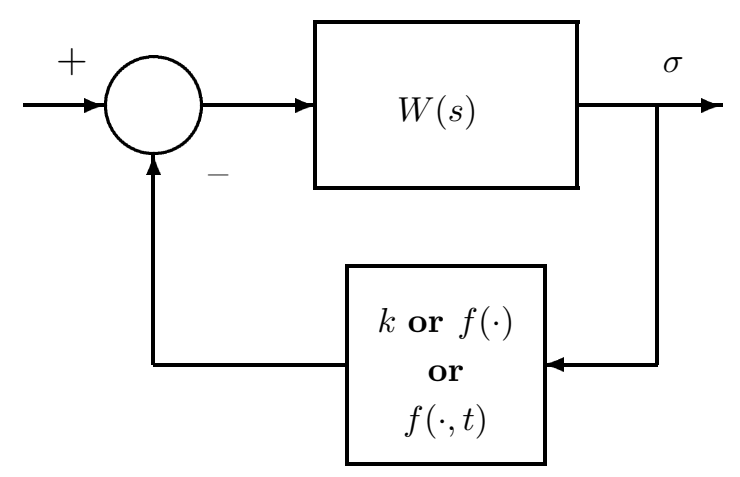

Figure 1: Closed-loop Linear/Nonlinear System

The Nyquist criterion states that the closed-loop system in Fig. 1 with $k$ in the feedback path will be stable if the point $-1 / k$ is not in the $W(s)$-map of the righthalf of the $s$-plane (RHP). In the case of a stable plant $W(s)$, this map is simply that region to the right as one traverses the Nyquist plot of $W(j \omega)$. In the case of a plant with $q$ poles in the right-half plane, this map must take into consideration $q$ map layers due to excluding 
these singularities by traversing a small circle around each in the counter-clockwise sense, which results in a large circle traversed in the clockwise sense for each. As an example, consider the unstable plant:

$$
W(s)=\frac{s+2}{s^{2}-4 s-5}
$$

Then the $s$-plane region being mapped is depicted in Fig. 2 and the resulting complete Nyquist diagram is

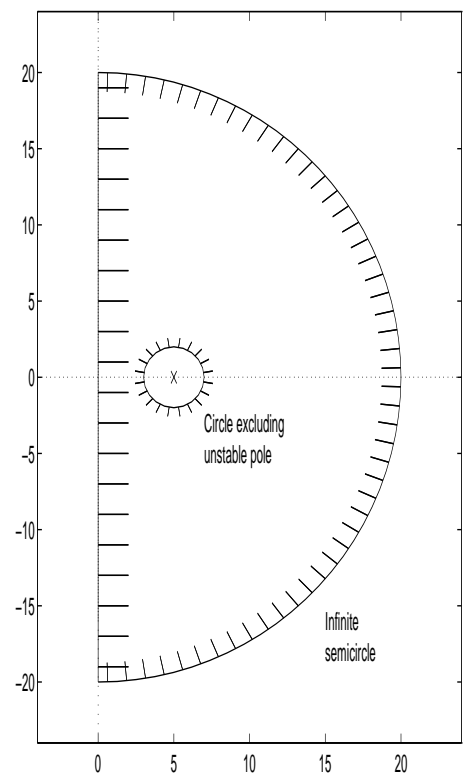

Figure 2: $s$-plane region mapped for Nyquist criterion

portrayed in Fig. 3. Observe that the only range of $k$ leading to stability is $4<k<\infty$; the corresponding region for $-1 / k$ is shown with the numeral 0 on the real axis to denote no closed-loop poles in the RHP. For $2.5<$ $k<4$ there will be 2 unstable closed-loop poles, and for the range $-\infty<k<2.5$ there will be one; again, the numerals on the real axis indicate these results.

The Circle Criterion $[1,3]$ states that the closed-loop system in Fig. 1 with $f(\cdot, t)$ in the sector $[\underline{F}, \bar{F}]$, i.e.,

$$
\underline{F} \leq \frac{f(\sigma, t)}{\sigma} \leq \bar{F}
$$

is absolutely stable (uniformly asymptotically stable in the large - UASIL) if one can draw a circle on the $W=U+j V$ plane whose diameter is defined by the points $V=0,-1 / \underline{F}<U<-1 / \bar{F}$ and whose interior has no points in common with the $W(s)$-map of the right-half of the $s$-plane. In essence, this circle must lie in a region of the Nyquist plane where there are zero mapping layers in the sense of the Nyquist criterion stated above. The case $\underline{F}<0<\bar{F}$ is interesting in that the "diameter" so defined passes through the point $\infty$ and the "inside" of the circle is actually what would

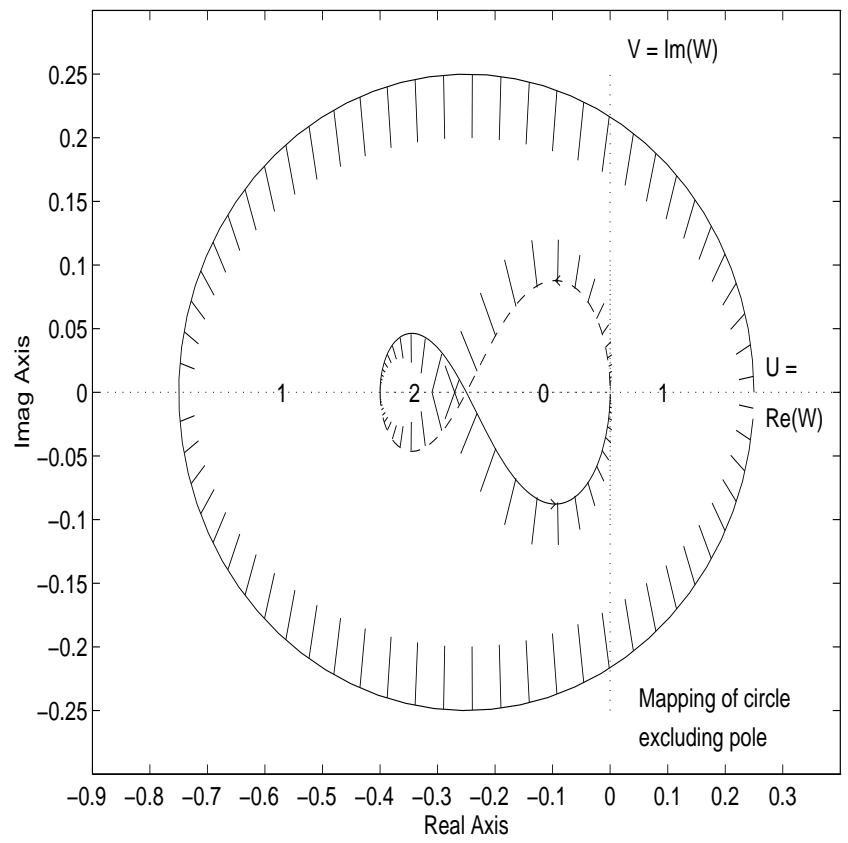

Figure 3: $W(s)$-map for the Nyquist criterion

ordinarily be considered the outside (exterior points), as shall be illustrated in the examples of Section 5 .

The Popov Criterion [2,3] states that the closed-loop system in Fig. 1 with $f(\cdot)$ in the sector $[0, \bar{F}]$, i.e.,

$$
0 \leq \frac{f(\sigma)}{\sigma} \leq \bar{F}
$$

is absolutely stable (UASIL) if one can draw a line on the Popov plot, i.e., polar plot of $\widehat{W}=U+j \widehat{V} \triangleq U+j \omega V$, passing through the point $-1 / \bar{F}$ such that the Popov locus of $W(j \omega)$ lies entirely to the right of the line.

Unfortunately, some of the elegance of the Circle Criterion is lost, due to this use of a modified frequencydomain plot. In particular, the general finite-sector case $[\underline{F}, \bar{F}]$ cannot be stated in graphical terms; rather, one must resort to the finite-sector transform [3] to convert the case $[\underline{F}, \bar{F}]$ to $[0,(\bar{F}-\underline{F})]$ by applying the above criterion to the transformed linear plant $\widetilde{W}$,

$$
\widetilde{W} \triangleq \frac{W}{1+\underline{F} W}
$$

which we do automatically in our tool ${ }^{1}$.

\footnotetext{
${ }^{1}$ While there is a Parabola Criterion for the general finitesector case [3], it is unattractive, due to it's being more strict than the Popov Criterion applied using the finite-sector transform.
} 


\section{Description of MATLAB Stabil- ity Routines}

Given the above problem definition, the following excerpts from the "help" displays constitute a concise "users' manual" for the routines newnyq, circle and popov:

$\operatorname{NEWNYQ}(A, B, C, D)$ produces a Nyquist plot. The frequency range, number of points and scaling are set automatically, and a "zoom" feature displays all real-axis crossings. In addition, the $k$ range(s) for closed-loop stability are reported, and the number of unstable closedloop poles for $-1 / k$ on various regions of the real axis are displayed.

NEWNYQ (A, B , C , D , 1) will cause the right-half plane mapping of $W(s)=C(s I-A) B+D$ to be indicated by hatching.

NEWNYQ (NUM, DEN) and NEWNYQ (NUM, DEN, 1) are corresponding variants for $W(s)$ provided in ratio of polynomial form.

CIRCLE (A, B, C, D, Fmin, Fmax) will check if the CC is satisfied for the sector-bounded nonlinearity and $W(s)=C(s I-A) B+D$

CIRCLE (A, B, C, D,Fmin) will determine the maximum value of $\mathrm{Fmax}(\bar{F})$ for which the $\mathrm{CC}$ is satisfied.

$\operatorname{CIRCLE}(A, B, C$, D , NaN , Fmax) will determine the minimum value of Fmin $(\underline{F})$ for which the $\mathrm{CC}$ is satisfied.

CIRCLE (NUM, DEN , Fmin, Fmax) et cetera are corresponding forms for $W(s)$ provided in ratio of polynomial form.

[Fmin, Fmax] =CIRCLE (A, B , C , D , Fmin) et cetera will return the sector bounds for which the $\mathrm{CC}$ is satisfied (even if, for example, Fmin,Fmax are supplied for which the CC is NOT OK).

The command summaries for popov are identical to those for circle.

\section{Algorithm Overview}

The Circle Criterion is implemented as follows: First, the finite-sector transform (Eqn. 6) is applied to reduce the criterion for UASIL to requiring that the Nyquist locus of $\widetilde{W}(j \omega)$ must lie to the right of a vertical line passing through $\widetilde{U}=-1 /(\bar{F}-\underline{F})$. (With a zero lower sector bound, the circle degenerates into a vertical line.) Then we analytically solve the polynomial equation corresponding to $d \widetilde{U}(j \omega) / d \omega=0$ using the root command, and thus locate all local minima and maxima of $\widetilde{U}(j \omega) \triangleq \operatorname{Re} \widetilde{W}(j \omega)$. These are inspected to determine the global minimum, i.e., the left-most point on the Nyquist locus of $\widetilde{W}(j \omega)$. Denoting the real part of this left-most point as $R_{\text {min }}$, the maximum upper sector bound permitted by the Circle Criterion is given by $\bar{F}=\underline{F}-1 / R_{\min }$. This maximum upper bound is reported to the user if only the lower bound is given (circle(num, den,Fmin)), or it is used to test the user's upper bound if it is also provided (circle (num, den, Fmin, Fmax)).

The algorithm for the Popov Criterion is also based on the analysis of $\widetilde{W}(j \omega)$. It is more complicated, since the Popov line may have an arbitrary orientation (angle with respect to the real axis). In essence, we define an auxiliary function $F(\theta)$ to be the difference between the two left-most points of the Popov locus rotated by the angle $\theta$ and use the MATLAB functionsolver fzero to find $\theta^{*}$ such that this difference is zero - this is the slope of the Popov line. Once the slope is known, the point where the Popov line crosses the real axis is readily determined; denoting this point as $R_{\text {min }}$, the maximum upper sector bound permitted by the Popov Criterion is again given by $\bar{F}=\underline{F}-1 / R_{\text {min }}$. This upper bound is reported to the user if only the lower bound is given (popov (num, den,Fmin)), or it is used to test the user's upper bound if it is also provided (popov (num, den, Fmin, Fmax)).

\section{$5 \quad$ Examples}

First we illustrate the use of newnyq and circle on a relatively simple stable plant:

$$
W(s)=\frac{s+1}{s^{4}+2 s^{3}+25 s^{2}+3 s+1}
$$

Note that the upper plot is similar to that produced by the MATLAB nyquist command except for the numerals on the real axis indicating the corresponding number of unstable closed-loop poles. The lower plot in Fig. 4 illustrates the feature of automatic zooming (to show all crossings of the real axis); the only manual operations in producing this figure were the text commands to document the problem (record num and den and the stable $k$ range). The report that newnyq provides after it is invoked for this problem is:

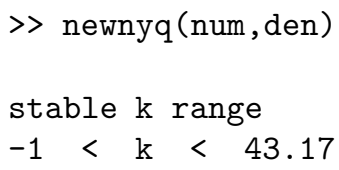



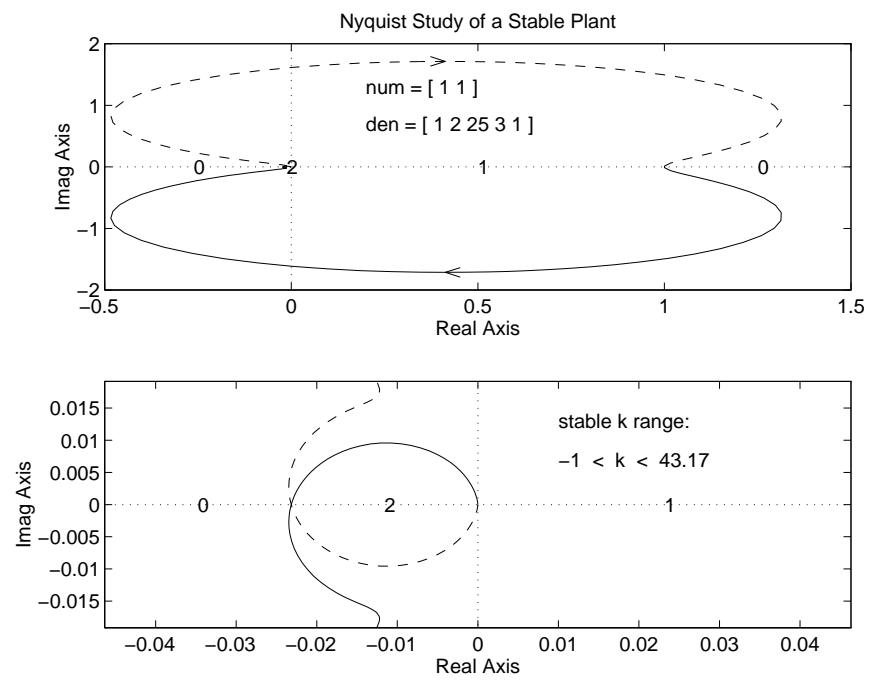

Figure 4: Nyquist Criterion Example (Stable Plant, with Zooming)

Finally, the numerals $0,1,2$ written on the real axis represent the number of right-half-plane closed-loop poles that would result if $-1 / k$ were to lie in each region.

Once the Nyquist plot has been viewed, one may request that the CC be applied to the same $W(j \omega)$ and a lower sector bound of $F_{\min }=2.5$. The CC locus is shown on

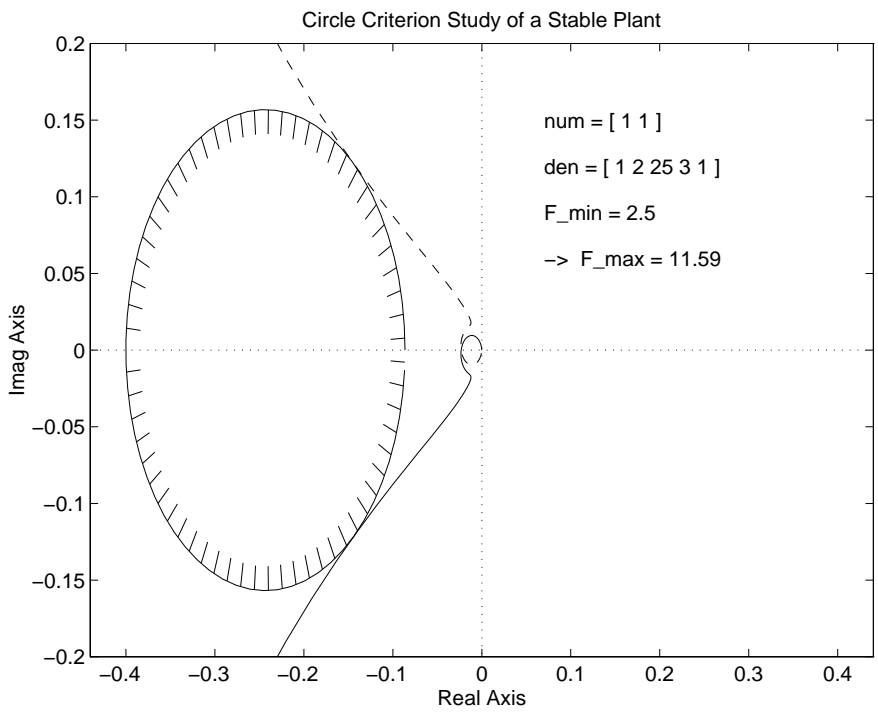

Figure 5: Circle Criterion Example (Stable Plant)

the Nyquist plot (Fig. 5), and the report that circle provides after it is invoked for this problem is:

> circle(num, den,2.5)

stable $\mathrm{k}$ range

$-1<\mathrm{k}<43.17$ circle criterion is satisfied

maximum sector bound F_max $=11.59$

(note that it includes the newnyq report, as shown above). Again - the only manual steps in preparing Fig. 5 were the documentation num $=$, den $=, F$ min $=$ etc. defining the problem and the result. The interpretation of this result is that $f(\cdot, t)$ in Fig. 1 must lie in the sector $[2.5,11.59]$, i.e.,

$$
2.5 \leq \frac{f(\sigma, t)}{\sigma} \leq 11.59
$$

in order to guarantee absolute stability.

We conclude this example by illustrating the observation in Section 2 that the "interior" of the CC circle may include the point $\infty$ by taking $F_{\text {min }}=-0.5$ for the above plant. The result is shown in the following report and Fig. 6:
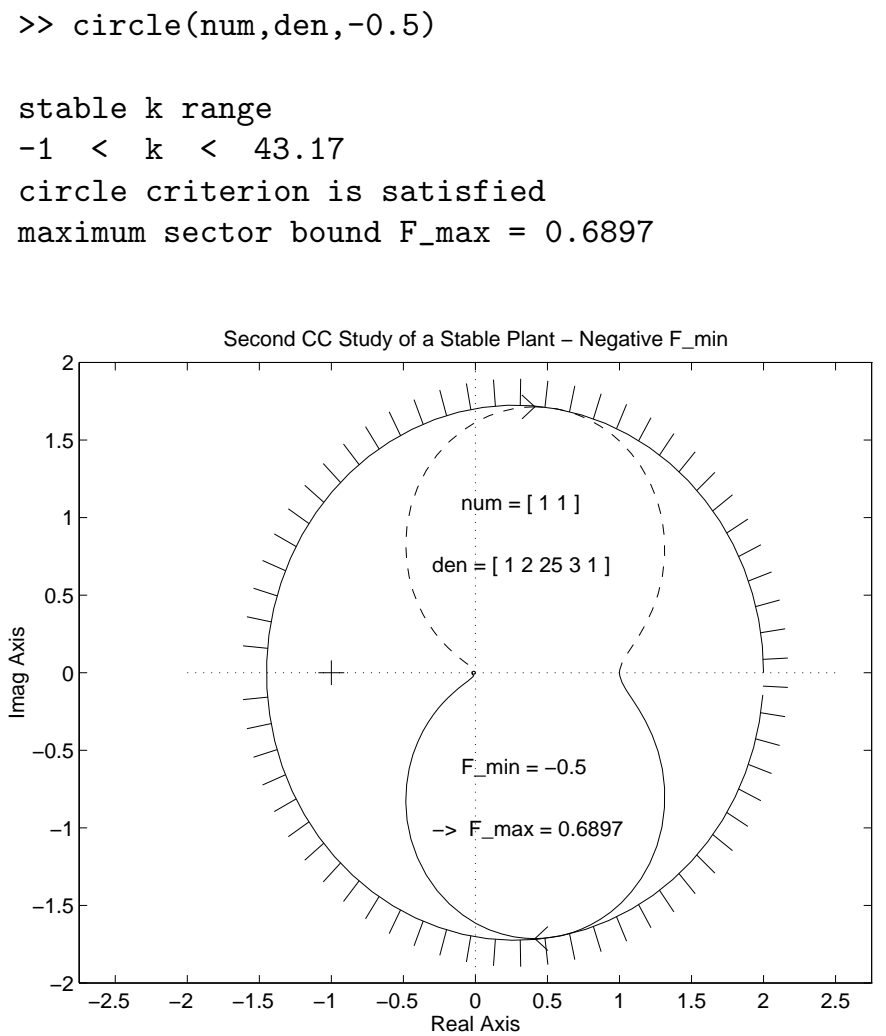

Figure 6: Circle Criterion Result for Negative F_min

Next, we provide the MATLAB plots obtained applying newnyq and circle to the unstable plant in Eqn. 3. The resulting "new Nyquist" plot is depicted in Fig. 7; note that it is similar to Fig. 3 except that the "large (infinite) circle" arising from the exclusion of the right-half-plane singularity is not shown (to avoid unnecessary clutter); that circle is, however, the basis for 


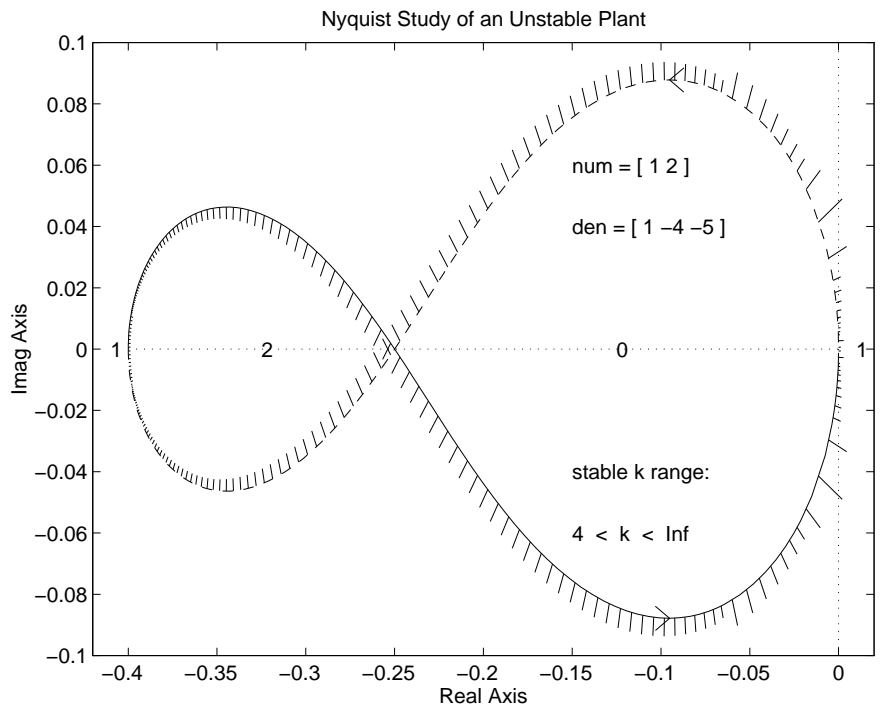

Figure 7: Nyquist Criterion Example (Unstable Plant)

the numeral 1 at the far left on the real axis (meaning that any $k$ such that $-1 / k<-0.4$ will result in a closed-loop system with one unstable pole). The region $-0.25<-1 / k<0$ on the real axis has the numeral 0 , because the points are to the left of the $W(j \omega)$ locus meaning that points there are no longer in the righthalf-plane map of $W(s)$. In light of this, the report that newnyq provides in this case is:

\section{>> newnyq (num, den)}

stable $\mathrm{k}$ range

$4<\mathrm{k}<$ Inf

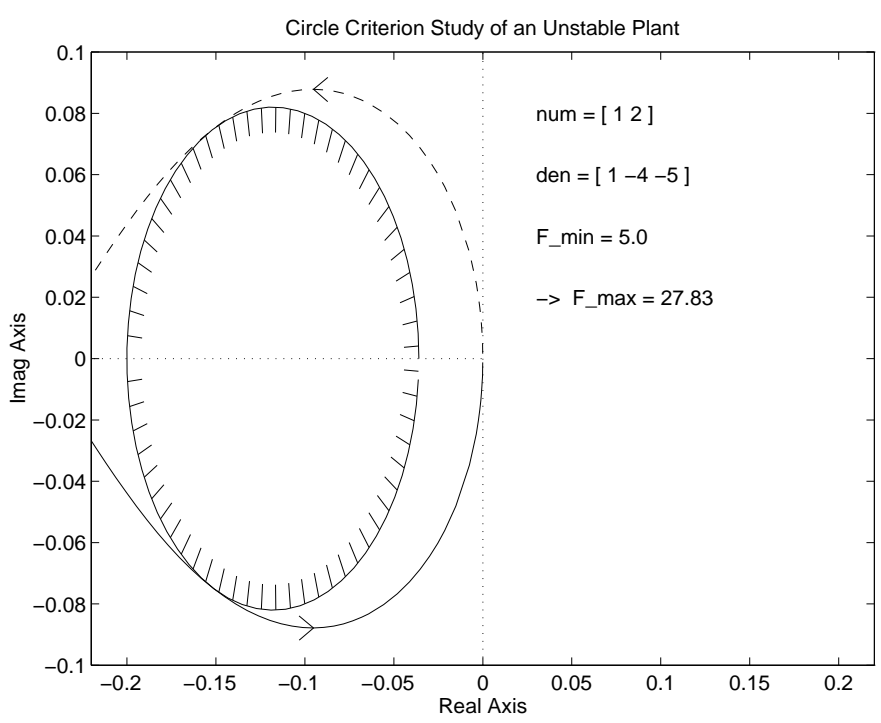

Figure 8: Circle Criterion Example (Unstable Plant)
To illustrate the application of the Circle Criterion tool to the same unstable plant, we specify a lower bound of $\underline{F}=5$, which is in the Nyquist range as required, and the report that circle provides for this problem is:

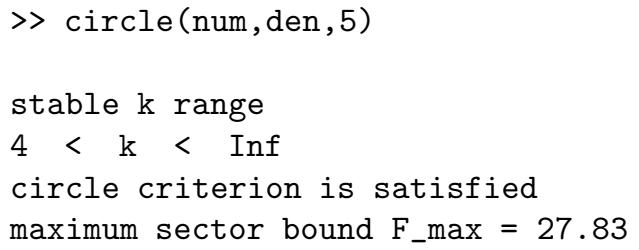

Again, we note that the corresponding circle in the Nyquist plane lies completely within the "no mapping" portion of the plot.

Another feature of circle allows us to specify the upper bound $\left(F_{\max }\right)$ and obtain the minimum corresponding lower bound that guarantees UASIL. To demonstrate this we issue the command circle (num, den, $\mathrm{NaN}, 100$ ) for the above unstable plant. We receive the following report and plot:

> circle (num, den, $\mathrm{NaN}, 100)$

stable $\mathrm{k}$ range

$4<\mathrm{k}<\operatorname{Inf}$

circle criterion is satisfied

minimum sector bound $F_{-}$min $=5.392$

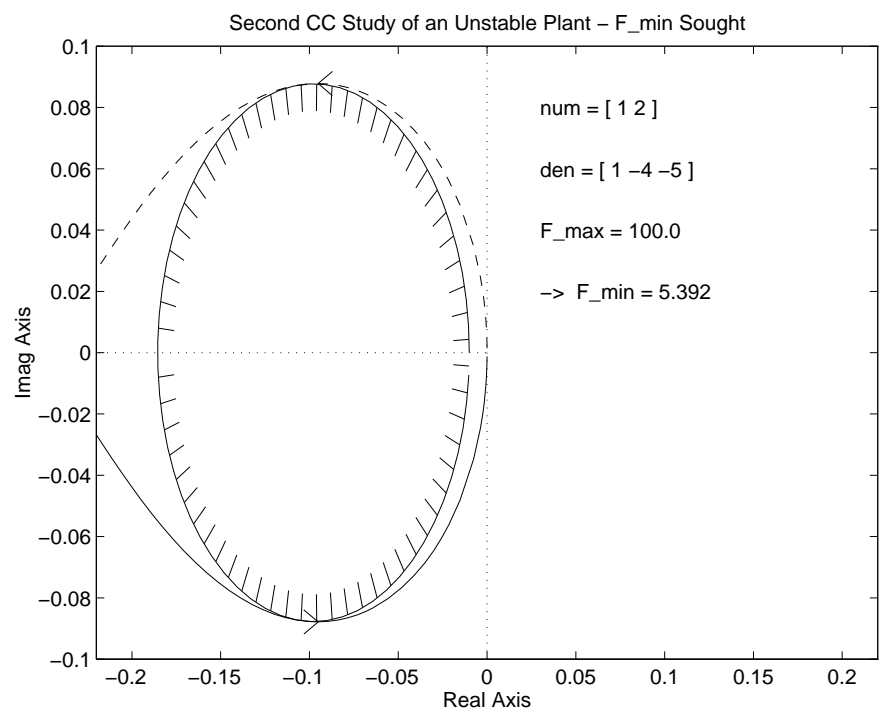

Figure 9: Circle Criterion Result, Upper Bound Given

Finally, we apply the Popov Criterion tool to the same stable plant $W(s)$ as before, first for the case when $\underline{F}=$ 0 , so the Popov locus is rendered for the same $W(j \omega)$ as shown in Fig. 4: The report for this case is as follows: 


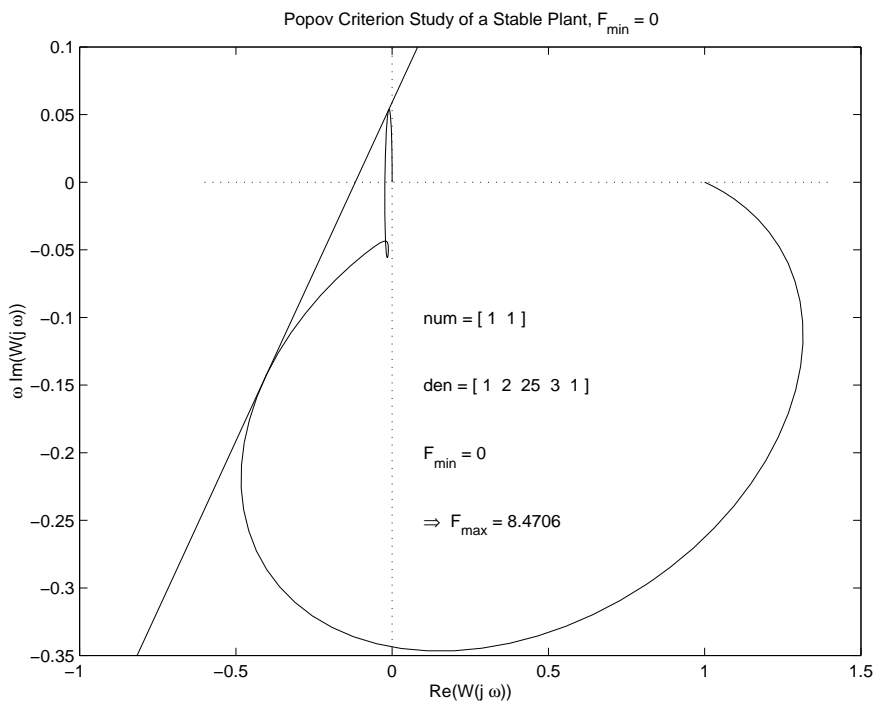

Figure 10: Popov Criterion Example (Stable Plant, $\underline{F}$ $=0)$

> popov(num, den, 0)

popov criterion is satisfied

minimum sector bound F_min $=0 \rightarrow$

maximum sector bound $F_{-} \max =8.4706$

Now, the same tool is applied to the case $\underline{F}=2.5$, which may be compared directly with the $\overline{C C}$ example depicted in Fig. 5: The report for this case is as

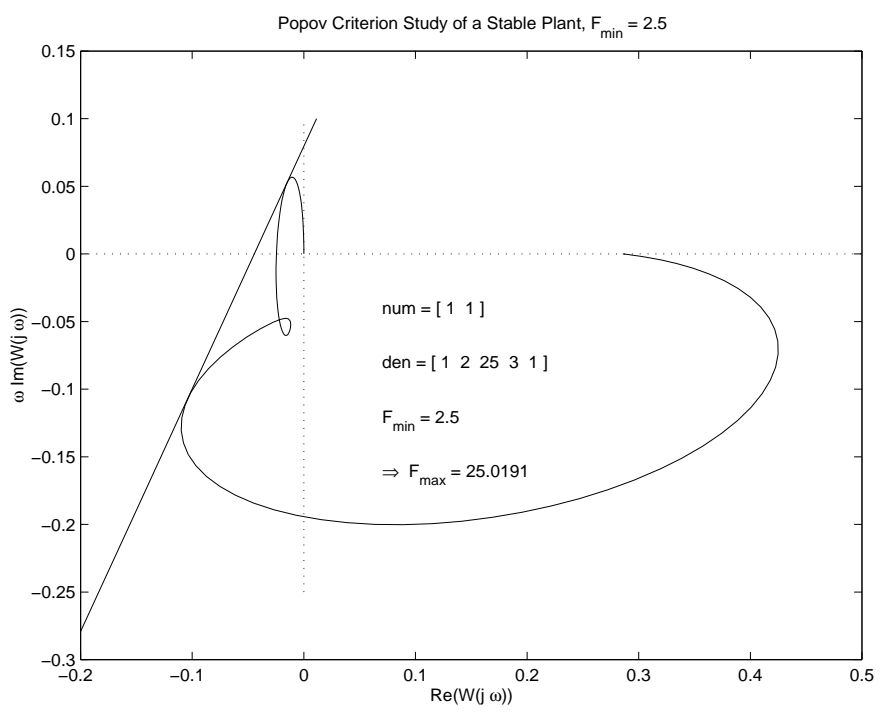

Figure 11: Popov Criterion Example (Stable Plant, $\underline{F}$ $=2.5$ )

follows:

>> popov (num, den, 2.5) popov criterion is satisfied

minimum sector bound $F_{-}$min $=2.5 \rightarrow$

maximum sector bound $F_{-} \max =25.019$

The $\mathrm{CC}$ gave the substantially smaller upper sector bound $\mathrm{F} \max =11.59$, which is not surprising considering that the CC guarantees UASIL for nonlinear timevarying systems while the Popov Criterion requires that $f$ be time invariant. In fact, the CC upper sector bound may be obtained by drawing a vertical line that just touches the Popov locus in Fig. 11, since the left-most extent of the Popov and Nyquist loci are the same since the abscissa for both plots is the same $(U=\operatorname{Re}[W(j \omega)])$.

\section{Conclusion}

These tools provide a simple environment for the determination of stability conditions for linear and nonlinear plants. The convenience and added support they supply can be readily appreciated from the examples presented in Section 4. We have installed these routines on our web page, URL $=$ http://www.ee.unb.ca/jtaylor/ for your access. A copy of this paper is also available there, to serve as a small "User's Guide".

\section{References}

[1] Narendra, K. S. and Goldwyn, R. M. "A Geometrical Criterion for the Stability of Certain Nonlinear Nonautonomous Systems", IEEE Trans. Circuit Theory, CT-11, No. 3, 1964.

[2] Popov, V. M., "Nouveaux Criteriums de Stabilité pour les Systemès Automatiques Non-Linéaries", Revue d'Electrotechnique et d'Energetique, Acad. de la Populaire Romaine, 5, No. 1, 1960.

[3] Narendra, K. S. and Taylor, J. H., Frequency Domain Criteria for Absolute Stability, Academic Press Inc., Reading, MA, 1973. 